\title{
Seepage Mechanism and Transient Pressure Analysis of Shale Gas*
}

\author{
Xiao Guo, Weifeng Wang \\ State Key Laboratory of Oil and Gas Reservoir Geology and Exploitation, Southwest Petroleum University, Nanchong, China \\ Email: guoxiao@swpu.edu.cn
}

Received October 9, 2012; revised November 9, 2012; accepted November 17, 2012

\begin{abstract}
The current research of nonlinear seepage theory of shale-gas reservoir is still in its infancy. According to the characteristics of shale gas in adsorption-desorption, diffusion, slippage and seepage during accumulation, migration and production, a mathematical model of unstable seepage in dual-porosity sealed shale-gas reservoir was developed while considering Knudsen diffusion, slip-flow effect and Langmuir desorption effect. By solving the model utilizing the Stehfest numerical inversion and computer programming in Laplace space, several typical curves of bottomhole pressure were obtained. In this paper, we discussed the effects of several parameters on the pressure dynamics, i.e. storativity ratio, Langmuir volume, Langmuir pressure, adsorption-desorption, tangential momentum accommodation coefficient, flow coefficient, boundary. The results show that the desorbed gas extends the time for fluid to flow from matrix system to fracture system; the changes of Langmuir volume and Langmuir pressure associated with desorption and adsorption effect are the internal causes of the storativity ratio change; when the tangential momentum accommodation coefficient decreases, the time for pressure wave to spread to the border reduces; interporosity flow coefficient determines the occurrence time of the transition stage; boundary range restricts the time for pressure wave to spread to the border.
\end{abstract}

Keywords: Shale Gas; Seepage Mechanism; Mathematical Model; Knudsen Diffusion; Slippage; Langmuir Desorption

\section{Introduction}

With the successful development of shale gas in North America and the sharp increase in demand of natural gas in China, Chinese government and petroleum companies are attaching more and more attention on shale gas development. Studying shale gas percolation mechanism is the foundation and premise of effective development. However, there are only a few articles on exploitation of shale gas. Besides, most scholars still use conventional theories and methods to study shale-gas reservoirs.

So far, some studies have been made in shale gas exploration. The idea of applying desorption theories of coalbed methane in shale-gas reservoir has been suggested several years ago [1]. And a capacity descending chart had been plotted by Duan Yonggang et al. (2011). In their study shale gas adsorption and desorption were considered [2].

In the past years, some scholars established a new gas well productivity formula. In their study, they considered the effect of artifici al fracturing effect and gas slippage effect. Besides, some of their researches were conducted by applying physical simulation [3].

\footnotetext{
*Special Issue-Numerical Analysis
}

To fully understand the mechanism of seepage in shale-gas reservoir, observation of nano-pores is dispensable. And for the first time, F. Javadpour (2009) used nanoscope to observe nano-pores in the shale-gas reservoir. He calculated the apparent Darcy permeability, while considering Knudsen diffusion. In addition, V. Shabr et al. (2011) introduced a new surface mass balance law to model transient desorption. In his research, he studied nanoscale seepage mechanism in pores, while considering Knudsen diffusion and Langmuir desorption.

In some studies, many prominent results were achieved, but on the other hand, Darcy's law was used [4,5]. In fact, in nanoscale pores, Darcy's law is no longer valid [6]. According to the characteristics of shale gas in adsorption-desorption, diffusion, slippage and seepage during accumulation, migration and production, a mathematical model of unstable seepage in dual-porosity confined shale-gas reservoir was built in this paper, while considering Knudsen diffusion, slip-flow effect and Langmuir desorption effect [7]. By solving the model utilizing the Stehfest numerical inversion and computer programming in Laplace space, several typical curves of bottomhole pressure were obtained [8-11]. And we discussed the effects of several parameters on the pressure dynamics, 
i.e. storativity ratio, Langmuir volume, Langmuir pressure, adsorption-desorption, tangential momentum accommodation coefficient, flow coefficient, boundary.

\section{Mathematical Model and Solutions}

\subsection{Assumptions}

To simplify the mathematical model, and make it convenient to solve, the following assumptions were made:

1) The shale-gas reservoir is dual-porosity;

2) The entire seepage process in shale-gas reservoir is isothermal;

3) Unsteady seepage in porous media doesn't follow Darcy law;

4) Flow in artificial fracture follows Darcy law;

5) The gas diffusi on flux in artificial fracture can be neglected when calculated with Darcy flow flux.

\subsection{Mathematical Model}

In nanoscale shale matrix pores, the mean free path of gas molecule is comparable or slightly less than pore diameter. The major forms of migration of shale gas in pores are Knudsen diffusion and slippage, while Knudsen diffusion inside the pore and slippage on its inner face [4,12-15].

Take a pore as example. Inside the pore, the mass flux of diffusing gas is described as following:

$$
V_{k}=\frac{2 r_{p o r o} M}{3 Z R_{g} T} \sqrt{\frac{8 R_{g} T}{\pi M}} \nabla p=\frac{M D_{k}}{Z R_{g} T} \nabla p
$$

where $D_{k}=\frac{2 r_{\text {poro }}}{3} \sqrt{\frac{8 R_{g} T}{\pi M}}$ is Knudsen diffusion coefficient [13].

On the inner face of the pore, the mass flux is described by

$$
V_{F}=\frac{\pi r_{\text {poro }} \rho}{8 \bar{p}}\left(\frac{2}{\alpha}-1\right) \sqrt{\frac{8 R_{g} T}{\pi M}} \nabla p=\frac{F r_{\text {poro }}^{2} \rho}{8 \mu} \nabla p .
$$

Where $F=\left(\frac{2}{\alpha}-1\right) \frac{\pi \bar{\mu}}{r_{\text {poro }} \bar{p}} \sqrt{\frac{8 R_{g} T}{\pi M}}$ is slippage coefficient [14]. Here, Darcy flow item is not considered.

Quantitative description of the relationship between shale gas adsorption and desorption under constant temperature is obtained by using Langmuir isothermal adsorption equation [15].

$$
V_{E}=V_{L} \frac{p}{p_{L}+p} .
$$

According to the mass conservation law and Langmuir isothermal adsorptio $\mathrm{n}$ equation and considering the Knudsen diffusion effect and slippage effect, the unsteady flow mathematical model of the dual-porosity sealed shale-gas reservoir is established. We assumed the matrix unit as spherical, and its diameter is $r_{1}$. In the center of the spherical matrix unit, we assumed the pressure is zero, and at the interface of matrix system and fracture system, the pressure equals to that of fracture system.

The seepage in fractures is described as

$$
\frac{1}{r} \frac{\partial}{\partial r}\left(\frac{p_{f}}{\mu Z} r \frac{\partial p_{f}}{\partial r}\right)=\frac{\phi_{f} p_{f} C_{f}}{K_{f} Z} \frac{\partial p_{f}}{\partial t}-\frac{R_{g} T}{M} \frac{q_{m}}{K_{f}}
$$

where $q_{m}$ is the generation term (the volume of gas that outflow from matrix unit).

$$
q_{m}=-\left.\frac{3}{r_{1}} \frac{\partial V_{k}}{\partial r}\right|_{r=r_{1}}=-\left.\frac{3}{r_{1}} \frac{M D_{k}}{Z R_{g} T} \frac{\partial p}{\partial r}\right|_{r=r_{1}} .
$$

The unsteady seepage in spherical matrix is described as

$$
\begin{aligned}
& \frac{1}{r^{2}} \frac{\partial}{\partial r}\left[r^{2}\left(\frac{\bar{\mu} D_{k}}{\bar{p}}+\frac{F r_{\text {poro }}^{2}}{8}\right) \frac{p}{\mu Z} \frac{\partial p_{m}}{\partial r}\right] \\
= & \phi_{m} \frac{p}{Z}\left(C_{m}+\frac{\rho_{g s c} V_{L} p_{L}}{\phi_{m} \rho_{m}\left(p_{L}+p_{m}\right)^{2}}\right) \frac{\partial p_{m}}{\partial t}
\end{aligned} .
$$

By substituting Equations (1)-(3) into the mass conservation law, we can get

$$
\begin{aligned}
& \frac{1}{r^{2}} \frac{\partial}{\partial r}\left[r^{2}\left(\frac{\bar{\mu} D_{k}}{\bar{p}}+\frac{F r_{\text {poro }}^{2}}{8}\right) \frac{p}{\mu Z} \frac{\partial p_{m}}{\partial r}\right] \\
= & \phi_{m} \frac{p}{Z}\left(C_{m}+\frac{\rho_{g s c} V_{L} p_{L}}{\phi_{m} \rho_{m}\left(p_{L}+p_{m}\right)^{2}}\right) \frac{\partial p_{m}}{\partial t}
\end{aligned}
$$

Boundary conditions:

$$
\left.\frac{\partial p_{m}}{\partial r}\right|_{r=0}=0 ;\left.p_{m}(r, t)\right|_{r=r_{1}}=p_{f}
$$

\section{Definitions}

For convenience, we define following variables pseudopressure $m=\frac{\mu_{i} Z_{i}}{p_{i}} \int \frac{p}{\mu Z} \mathrm{~d} p$;

dimensionless pseudopressure $m_{D}=\frac{2 \pi K_{f} h\left(m_{i}-m\right)}{q_{s c} B_{i} \mu_{i}}$;

dimensionless time $t_{D}=\frac{K_{f} t}{\left(V \phi C_{t}\right)_{f+m} \mu_{i} r_{w}^{2}}$;

dimensionless diameter of fracture $r_{f D}=\frac{r}{r_{w}}$;

dimensionless diameter of matrix $r_{m D}=\frac{r}{r_{1}}$;

interporosity flow coefficient 


$$
\lambda=\frac{15 r_{w}^{2}}{r_{1}^{2}} \frac{K_{a p p}}{K_{f}}=\frac{15 r_{w}^{2}}{r_{1}^{2} K_{f}}\left[\frac{\bar{\mu} D_{k}}{\bar{p}}+\frac{F r_{\text {poro }}^{2}}{8}\right]
$$

matrix apparent permeability

$$
k_{\text {app }}=\frac{\bar{\mu} D_{k}}{\bar{p}}+\frac{F r_{\text {poro }}^{2}}{8}=\left[\frac{2}{3}+\frac{\pi}{8}\left(\frac{2}{\alpha}-1\right)\right] \frac{r_{\text {poro }} \bar{\mu}}{\bar{p}} \sqrt{\frac{8 R_{g} T}{\pi M}}
$$

matrix total compressibility coefficient

$$
C_{t m}=C_{m}+\frac{\rho_{g s c} V_{L} p_{L}}{\phi_{m} \rho_{m}\left(p_{L}+p_{m}\right)^{2}}
$$

elastic storativity ratio

$$
\omega=\frac{\left(V \phi C_{t}\right)_{f}}{\left(V \phi C_{t}\right)_{f+m}}=\frac{\phi_{f} C_{t f}}{\phi_{f} C_{t f}+\phi_{f}\left[C_{m}+\frac{\rho_{g s c} V_{L} p_{L}}{\phi_{m} \rho_{m}\left(p_{L}+p_{m}\right)^{2}}\right]}
$$

Introduce pseudopressure in Laplace space, and then the unsteady seepage in spherical matrix and the boundary conditions can be described as

$$
\begin{gathered}
\frac{\partial^{2} \bar{m}_{D}}{\partial r_{m D}^{2}}+\frac{2}{r_{m D}} \frac{\partial \bar{m}_{D}}{\partial r_{m D}}=W^{2} \bar{m}_{D} \\
\left.\frac{\partial \bar{m}_{D}}{\partial r_{m D}}\right|_{r_{m D}=0}=0 \\
\left.\bar{m}_{D}\left(r_{D}, s\right)\right|_{r_{m D}=1}=\bar{m}_{f D}
\end{gathered}
$$

where

$$
W^{2}=\frac{15(1-\omega) s}{\lambda} .
$$

$s$ is Laplace variable.

By substituting Equations (10) and (11) into Equation (9), pressure between spherical matrix and fracture can be described as

$$
\left.\frac{\partial \bar{m}_{D}}{\partial r_{m D}}\right|_{r_{m D}=1}=(W \operatorname{coth} W-1) \bar{m}_{f D} .
$$

Transform Equation (4) into dimensionless equation and make Laplace transformation, and then we can describe seepage in fracture as:

$$
\begin{aligned}
& \frac{\partial^{2} \bar{m}_{f D}}{\partial r_{f D}^{2}}+\frac{1}{r_{f D}} \frac{\partial \bar{m}_{f D}}{\partial r_{f D}} \\
= & \omega s \bar{m}_{f D}+\left.\frac{\lambda}{5+\frac{15 \pi}{16}\left(\frac{2}{\alpha}-1\right)} \frac{\partial \bar{m}_{D}}{\partial r_{m D}}\right|_{r_{m D}}=1
\end{aligned}
$$

By substituting Equation (13) into Equation (14), coupled equation of fluid seepage in fractures and diffusion and slippage in matrix is deduced:

$$
\frac{\partial^{2} \bar{m}_{f D}}{\partial r_{f D}^{2}}+\frac{1}{r_{f D}} \frac{\partial \bar{m}_{f D}}{\partial r_{f D}}=f(s) \bar{m}_{f D}
$$

where the interporosity flow function is

$$
\begin{aligned}
f(s) & =\omega s+\frac{\lambda}{5+\frac{15 \pi}{16}\left(\frac{2}{\alpha}-1\right)} \\
& \left(\sqrt{\frac{15(1-\omega) s}{\lambda}} \operatorname{coth} \sqrt{\frac{15(1-\omega) s}{\lambda}}-1\right)
\end{aligned}
$$

\subsection{Solutions}

Provided that there is a vertical well in the sealed dualporosity shale-gas reservoir, the boundary conditions corresponding with pseudopressure in Laplace space are as follows(consider wellbore storage effect $C_{D}$ and skin factor $S$ ):

$$
\begin{gathered}
{\left[C_{D} s \bar{m}_{w D}-r_{D} \frac{\partial \bar{m}_{f D}}{\partial r_{f D}}\right]_{r_{f D}=1}=\frac{1}{S}} \\
\left.\frac{\partial \bar{m}_{f D}}{\partial r_{f D}}\right|_{r_{f D}=R_{D}}=0 \\
\bar{m}_{w D}=\left[\bar{m}_{f D}-S \frac{\partial \bar{m}_{f D}}{\partial r_{f D}}\right]_{r_{f D}=1} .
\end{gathered}
$$

Equation (15) is the generalized Bessel equation, its general solution is

$$
\begin{aligned}
\bar{m}_{f D}= & A K_{0}\left(\sqrt{f(s)} r_{f D}\right) \\
& +B I_{0}\left(\sqrt{f(s)} r_{f D}\right)
\end{aligned}
$$

Substitute Equations (17) and (18) into Equation (20), and then factor $A$ can be calculated: (see Equation (21))

$$
B=M A \text {. }
$$

As to circle sealed formation

$$
M=\frac{K_{1}\left(\sqrt{f(s)} R_{D}\right)}{I_{1}\left(\sqrt{f(s)} R_{D}\right)}
$$

$$
A=\frac{1}{s} \cdot \frac{1}{C_{D} s\left[K_{0}(\sqrt{f(s)})+M I_{0}(\sqrt{f(s)})\right]+\left(C_{D} S s+1\right)\left[K_{1}(\sqrt{f(s)})-M I_{1}(\sqrt{f(s)})\right] \sqrt{f(s)}}
$$


Substitute $A$ and $B$ into Equation (20), then the distribution of pseudopressure in Laplace space can be described as (see Equation (24)).

Substitute Equation (24) and its derivation function into Equation (19), the unsteady pseudopressure (dimensionless) can be calculated, while considering $C_{D}$ and $S$. (see Equation (25)).

\section{Typical Curves and Analysis}

By applying Stehfest numerical inversion on the pseudopressure in Equation (13) in Laplace space, the relationship between $m_{w D}\left(t_{D}\right)$ and $t_{D} / C_{D}$ is obtained. According to Equation (13), main parameters are $\omega, V_{L}, P_{L}$, $\alpha, \lambda, R_{D}$, set $C_{D}=0.8, S=1.2$.

The effects of each parameter on pressure dynamics will be discussed next.

Figure 1 shows that the elastic storativity ratio $\omega$ determines the width and depth of the concavity in the shale gas pressure derivative curve. In another words, the smaller the storativity ratio, the longer the transition time and the lower the pressure.

Figure 2 indicates that the influence of Langmuir volume on the transition time for fluid to flow from matrix system to fracture system. Under the same Langmuir pressure, with the Langmuir volume increasing, the concavity in the curve is deepened. When the Langmuir volume increases to a certain degree, the concavity amplitude will reduce.

Figure 3 shows the influence of Langmuir pressure on the transition stage (presents the stage when interporosity fluid flow from matrix system to fracture system). Under the same Langmuir volume, with the Langmuir pressure increasing, the concavity is deepened. But when the Langmuir pressure increases to a certain degree, the concavity amplitude will reduce.
Figure 4 shows that the shale gas desorbed from matrix system enlarges the storage capacity of the matrix system, and prolongs the occurrence of the transition stage. It is also the reason why the storativity ratio decreases and the concavity deepens.

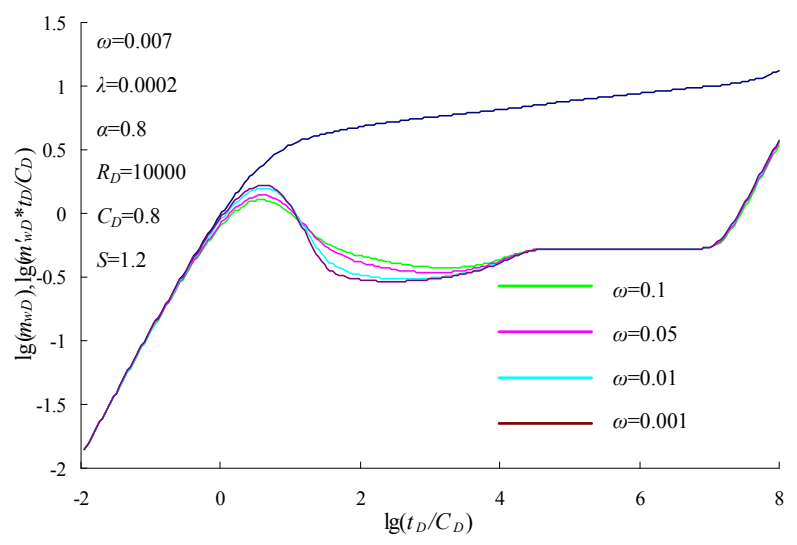

Figure 1. Effect of storativity ratio on pressure dynamics.

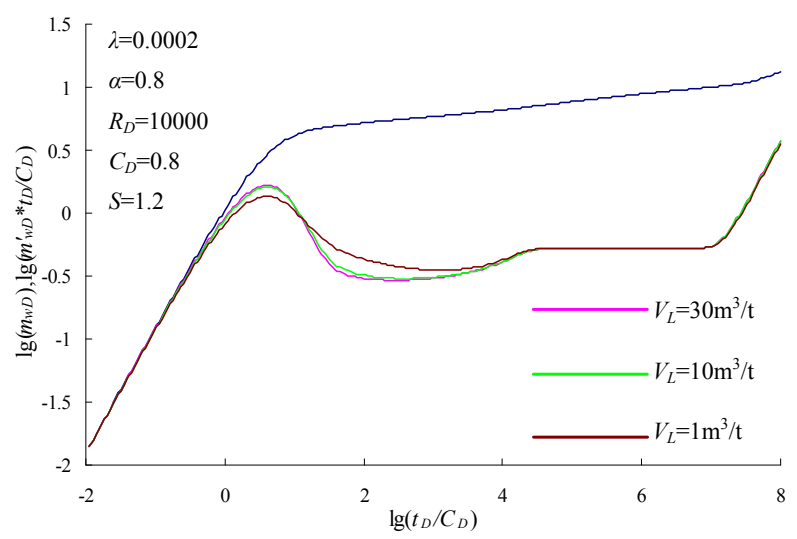

Figure 2. Effect of Langmuir volume on pressure dynamics.

$$
\begin{aligned}
& \bar{m}_{f D}\left(r_{f D}, s\right) \\
= & \frac{1}{s} \cdot \frac{K_{0}\left(\sqrt{f(s)} r_{D}\right)+\frac{K_{1}\left(\sqrt{f(s)} R_{D}\right)}{I_{1}\left(\sqrt{f(s)} R_{D}\right)} I_{0}\left(\sqrt{f(s)} r_{D}\right)}{C_{D} s\left[K_{0}(\sqrt{f(s)})+\frac{K_{1}\left(\sqrt{f(s)} R_{D}\right)}{I_{1}\left(\sqrt{f(s)} R_{D}\right)} I_{0}(\sqrt{f(s)})\right]+\left(C_{D} S s+1\right) \sqrt{f(s)}\left[K_{1}(\sqrt{f(s)})-\frac{K_{1}\left(\sqrt{f(s)} R_{D}\right)}{I_{1}\left(\sqrt{f(s)} R_{D}\right)} I_{1}(\sqrt{f(s)})\right]} \\
& \bar{m}_{w D}(s) \\
= & \frac{1}{s} \cdot \frac{K_{D} s\left[K_{0}(\sqrt{f(s)})+\frac{K_{1}\left(\sqrt{f(s)} R_{D}\right)}{I_{1}\left(\sqrt{f(s)} R_{D}\right)} I_{0}(\sqrt{f(s)})\right]+\left(C_{D} S s+1\right) \sqrt{f(s)}\left[K_{1}(\sqrt{f(s)})-\frac{K_{1}\left(\sqrt{f(s)} R_{D}\right)}{I_{1}\left(\sqrt{f(s)} R_{D}\right)} I_{1}(\sqrt{f(s)})\right.}{I_{1}\left(\sqrt{f(s)} R_{D}\right)} I_{0}(\sqrt{f(s)})+S \sqrt{f(s)}\left[K_{1}(\sqrt{f(s)})-\frac{K_{1}\left(\sqrt{f(s)} R_{D}\right)}{I_{1}\left(\sqrt{f(s)} R_{D}\right)} I_{1}(\sqrt{f(s)})\right]
\end{aligned}
$$




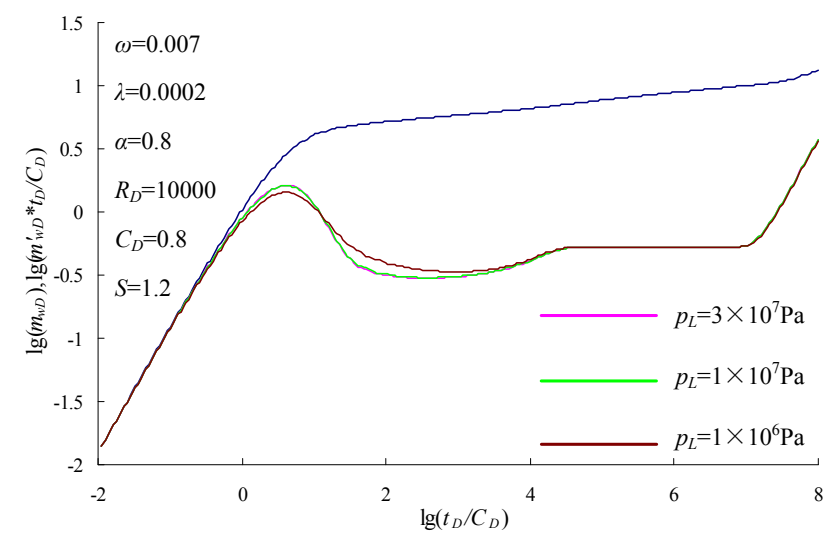

Figure 3. Effect of Langmuir pressure on pressure dynamics.

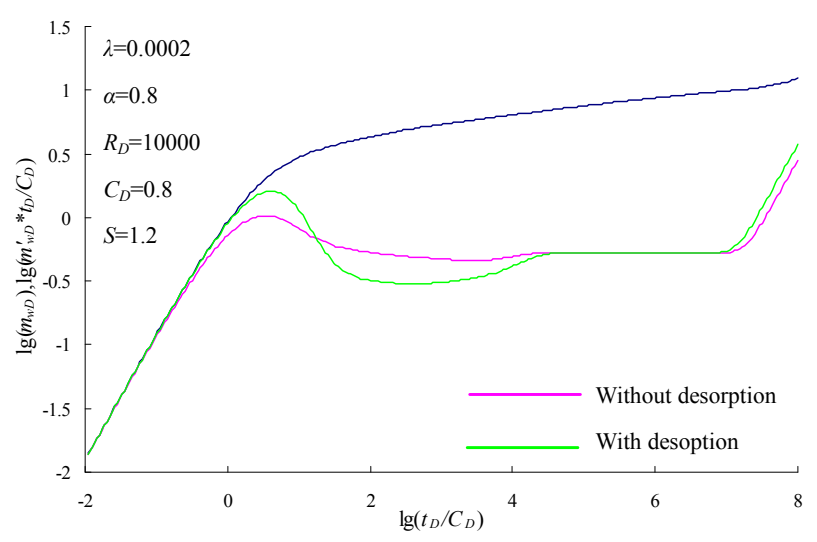

Figure 4. Effect of gas desorption on pressure dynamics.

Figure 5 describes the influence of the tangential momentum accommodation coefficient upon the pressure dynamics. With the tangential momentum accommodation coefficient decreasing, the concavity in the typical curve deviates to right and becomes shallower, and the time for pressure wave to spread to the border reduces.

Figure 6 shows that the interporosity flow coefficient determines the occurrence time of the transition stage too. With the interporosity flow coefficient increasing, the transition stage appears earlier, the concavity deviates to left and the interporosity flow gets more intense.

Figure 7 reflects how boundary range influences the pressure dynamics. With the range getting narrower, the time for pressure wave to spread to the border reduces. When the pressure wave reaches the boundary, the pressure curve as well as its derivative curve upturns, finally the two curves are tangent into a straight line, whose slope turn to be 1 .

From all the figures, we can conclude that the pressure change of unsteady seepage in vertical wells in sealed shale-gas reservoir can be divided into three stages:

- Early wellbore storage;

- Transition stage: interporosity flow (from matrix system to fracture system) appears. Concavity of the

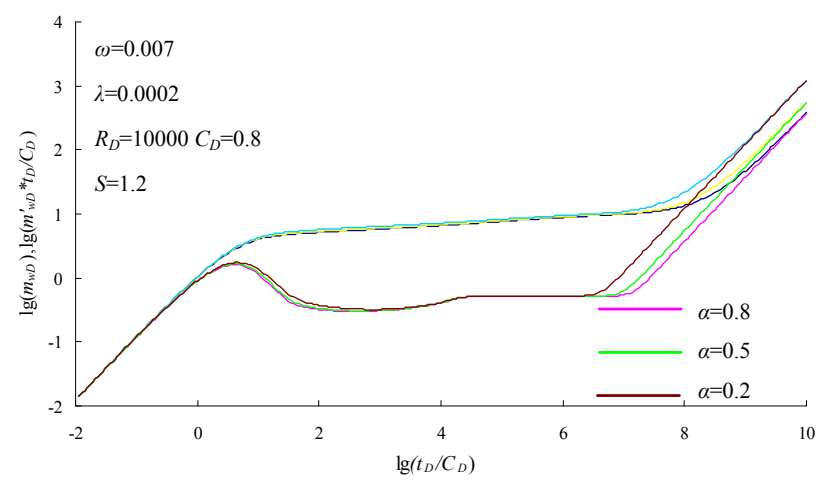

Figure 5. Effect of tangential momentum accommodation coefficient on pressure dynamics.

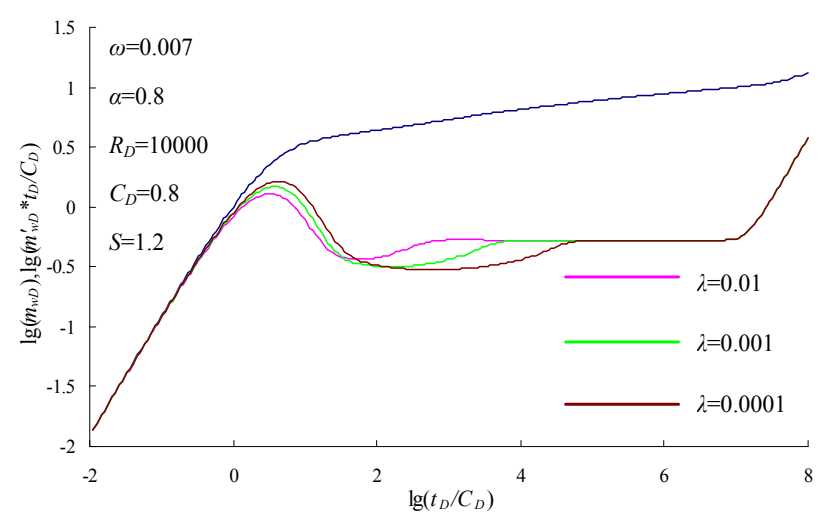

Figure 6. Effect of interporosity flow coefficient on pressure dynamics.

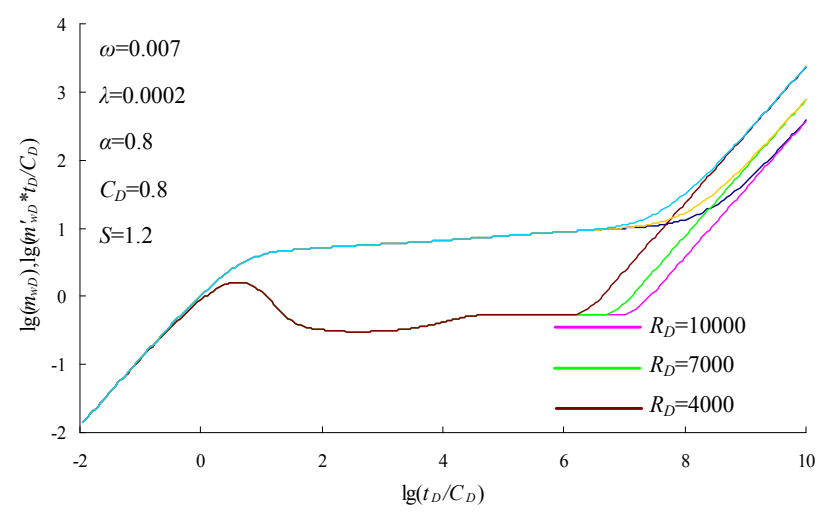

Figure 7. Effect of boundary on pressure dynamics.

curve appears in this stage too;

- The radial flow of dual porosity system: this stage presents the homogeneous characteristics of formation. Pressure derivative curve shows a horizontal line, whose value is 0.5 .

\section{Conclusions}

1) According to the characteristics of shale gas in adsorption-desorption, diffusion, slippage and seepage during accumulation, migration and production, a mathe- 
matical model of unsteady seepage in dual-porosity sealed shale-gas reservoir was built while considering Knudsen diffusion, slip-flow effect and Langmuir desorption effect. By solving the model utilizing the Stehfest numerical inversion and computer programming in Laplace space, several typical curves of bottomhole pressure were obtained.

2) In the sealed shale-gas reservoir, the stage when flow in fracture system exists only is extremely transient, the transition stage appears immediately after the wellbore storage stage. So in fracture system, radial flow doesn't appear. The pressure dynamics of unsteady seepage appear only in storage stage, the radial flow stage, and the stage when the pressure is unsteady.

3) The typical curves of bottomhole pressure are presented to discuss the influences of several sensitive parameters upon pressure behavior. These sensitive parameters include elastic storativity ratio, Langmuir volume, Langmuir pressure, adsorption-desorption, tangential momentum accommodation coefficient, interporosity flow coefficient, and boundary range. The smaller the storativity ratio, the longer the transition stage. The changes of Langmuir volume and Langmuir pressure, as well as desorption and adsorption mechanisms are the internal causes of the storativity ratio change. The tangential momentum accommodation coefficient describes smoothness of the pores' inner face, With the tangential momentum accommodation coefficient decreasing, the concavity of typical curves deviates right and becomes shallower, and the time for pressure wave to spread to the border reduces; The interporosity flow coefficient determines the occurrence time of the transition stage. With the interporosity flow coefficient increasing, the transition stage appears earlier, the concavity deviates to left and the interporosity flow gets more intense; with the boundary range getting narrower, the time for pressure wave to spread the border reduces.

\section{REFERENCES}

[1] Z. L. Ping and P. R. Fang, "Accumulation and Transformation of Shale Gas Reservoir," China Petroleum Exploration, Vol. 3, 2009, pp. 20-23.

[2] D. Y. Gang, W. M. Qiang and L. J. Qiu, "Shale Gas Seepage Mechanism and Fractured Wells Production Evaluation," Journal of Chongqing University, Vol. 34, No. 4, 2011, pp. 62-66.

[3] G. S. Sheng, Y. X. He and L. H. Xun, "Impact of Slippage on Shale Gas Well Productivity," Natural Gas
Tndustry, Vol. 31, No. 4, 2011, pp. 55-58.

[4] F. Javadpour, "Nanopores and Apparent Permeability of Gas Flow in Mudrocks (Shales and Siltstone)," Journal of Canadian Petroleum Technology, Vol. 48, No. 8, 2009, pp. 16-21. doi:10.2118/09-08-16-DA

[5] V. Shabro, C. Torres-Verdín and F. Javadpour, "Numerical Simulation of Shale-Gas Production: From Pore-Scale Modeling of Slip-Flow, Knudsen Diffusion, and Langmuir Desorption to Reservoir Modeling of Compressible Fluid," SPE North American Unconventional Gas Conference and Exhibition in The Woodlands, Texas, 14-16 June 2011, pp. 2-4. doi:10.2118/144355-MS

[6] D. Tzoulaki, L. Heinke and H. Lim. "Assessing Surface Permeabilities from Transient Guest Profiles in Nanoporous Host Materials," Angewandte Chemie International Edition, Vol. 48, No. 19, 2009, pp. 3525-3528. doi:10.1002/anie.200804785

[7] M. H. M. Hassan and J. D. Way, "Gas Transport in A Microporous Silica Membrane," SPE Abu Dhabi International Petroleum Exhibition and Conference, Abu Dhabi, 13-16 October 1996, pp. 2-3. doi:10.2118/36226-MS

[8] E. Ozkan and R. Raghavan, "Modeling of Fluid Transfer From Shale Matrix to Fracture Network," SPE Annual Technical Conference and Exhibition, Florence, 19-22 September 2010, pp. 10-13. doi:10.2118/134830-MS

[9] C. M. Freeman, G. Moridis, D. Ilk and T. A. Blasingame. "A Numerical Study of Performance for Tight Gas and Shale Gas Reservoir Systems," SPE Annual Technical Conference and Exhibition, New Orleans, 4-7 October 2009, pp. 9-12. doi:10.2118/124961-MS

[10] G. J. Moridis, T. A. Blasingame and C. M. Freeman, "Analysis of Mechanisms of Flow in Fractured Tight-Gas and Shale-Gas Reservoirs," SPE Latin American \& Caribbean Petroleum Engineering Conference Lima, Peru, 1-3 December 2010, pp. 20-22. doi:10.2118/139250-MS

[11] H. Stehfest, "Algorithm 368 Numerical Inversion of Laplace Transforms," Communications of ACM, Vol.13, No.1, 1970, pp. 47-49. doi:10.1145/361953.361969

[12] F. Javadpour, D. Fisher and M. Unsworth, "Nanoscale Gas Flow in Shale Gas Sediments," Canadian Petroleum Technology, Vol. 46, No. 10, 2007, pp. 55-61.

[13] S. Roy, R. Raju and F. H. Chuang, "Modelling Gas Flow through Microchannels and Nanopores," Journal of Applied Physics, Vol. 93, No. 8, 2003, pp. 4870-4879. doi:10.1063/1.1559936

[14] P. G. Brwon, A. Dinnado and K. G. Cheng, "The Flow of Gas in Pipes at Low Pressures," Journal of Applied Physics, Vol. 17, No. 10, 1946, pp. 802-813. doi:10.1063/1.1707647

[15] A. E. Schrodinger, "The Physics of Flow through Porous Media," University of Toronto Press, Toronto, 1974. 


\section{Nomenclature}

$V_{E}$ : total volume of gas adsorbed of per unit volume of the reservoir in equilibrium at pressure $P, \mathrm{~m}^{3} / \mathrm{t}$;

$V_{L}$ : Langmuir volume, the maximum sorption capacity of the shale, $\mathrm{m}^{3} / \mathrm{t}$;

$P_{L}$ : Langmuir pressure, at which the total gas volume adsorbed, $V_{E}$, is equal to $50 \%$ of the Langmuir volume, $\mathrm{Pa}$;

$P$ : pressure, $\mathrm{Pa}$;

$P_{i}:$ initial pressure, $\mathrm{Pa}$;

$\bar{p}$ : average pressure, $\mathrm{Pa}$;

$\omega$ : dimensionless storativity ratio;

$\lambda$ : dimensionless interporosity flow coefficient;

$K$ : absolute permeability, $\mathrm{m}^{2}$;

$M$ : molar mass, $\mathrm{kg} / \mathrm{mol}$;

$Z$ : gas compressibility, fraction;

$R_{g}$ : universal gas constant, $8.314 \mathrm{~Pa} \cdot \mathrm{m}^{3} /(\mathrm{mol} \cdot \mathrm{K})$;

$T$ : temperature, $\mathrm{K}$;

$\rho$ : density, $\mathrm{kg} / \mathrm{m}^{3}$

$\mu$ : viscosity, $\mathrm{Pa} \cdot \mathrm{s}$;

$\bar{\mu}$ : average viscosity $\mathrm{Pa} \cdot \mathrm{s}$;

$\varphi$ : porosity, fraction;
$C$ : gas compressibility, $\mathrm{Pa}^{-1}$;

$\alpha$ : tangential momentum accommodation coefficient, depending on the smoothness of the pores' inner face, gas type, temperature and pressure, fraction $(0 \sim 1)$;

$r$ : radial distance in spherical coordinates, $\mathrm{m}$;

$r_{\text {poro }}$ : matrix system pore radius, $\mathrm{m}$;

$r_{1}$ : spherical matrix block radius, $\mathrm{m}$;

$r_{w}$ : gas well radius, $\mathrm{m}$;

$R$ : gas reservoir boundary, $\mathrm{m}$;

$S$ : skin coefficient, fraction;

$C_{D}$ : dimensionless wellbore storage factor;

$m$ : pseudopressure;

$m_{D}$ : dimensionless pseudopressure;

$\bar{m}_{D}$ : dimensionless pseudopressure in Laplace space;

$\bar{m}_{w D}$ : dimensionless bottom hole pseudopressure in

Laplace space;

$s$ : Laplace transform variable;

$f(s)$ : interporosity flow function;

$I_{v}: v$ order of first species modified Bessel function,

$K_{v}: v$ order of second species modified Bessel function, $v=0.1$. 